\title{
Privileging Long-life Packets in Multihop Wireless Networks
}

\author{
Miguel Elias M. Campista, Luís Henrique M. K. Costa, and Otto Carlos M. B. Duarte \\ UFRJ - PEE/COPPE/GTA - DEL/POLI \\ P.O. Box 68504 - 21945-970, Rio de Janeiro, RJ, Brazil \\ E-mails:\{miguel,luish,otto\}@gta.ufrj.br
}

\begin{abstract}
The typical application of wireless mesh networks is Internet access. This application introduces a gateway, which becomes the preferred destination in the network. In such topology, the throughput share is unfair because the number of medium accesses increases with the distance to the gateway. In this work, we propose the Route-Length-based Fairness (RLF) mechanism to assign a higher priority on medium access for packets that traverse a high number of hops, named long-life packets. When comparing the proposed mechanism to IEEE 802.11g, the simulation results show a considerable improvement in fairness for applications running over UDP and TCP.

Keywords: Multihop wireless networks, wireless mesh networks, and fairness.
\end{abstract}

\section{INTRODUCTION}

Today, there is an increasing demand for ubiquitous connectivity. Wireless networks adequately address this need given the diffusion nature of radio-frequency transmissions, which provides interconnection flexibility, easy access, and mobility support to users. Furthermore, the reduction of the wired infrastructure makes the wireless technology a cost-effective solution for last-mile networks [1].

Wireless networks based on the IEEE 802.11 standard operate in infrastructure or ad hoc mode. In infrastructure mode, the architecture is centralized and communications are only possible within the range of access points. In ad hoc mode, on the other hand, the network is distributed and stations rely on multihop communications. The ad hoc mode, however, cannot guarantee connectivity since it depends on the collaboration of other nodes to deliver data. Wireless Mesh Networks combine the advantages of both modes. These networks extend the range of access points using a wireless backbone. This backbone is an ad hoc network in charge of guaranteeing connectivity to all wireless nodes.

A typical application of wireless mesh networks is to provide Internet access. In this scenario, one or more routers play the role of gateways to the wired infrastructure. One key problem is the throughput unfairness caused by the different number of hops a packet may have to traverse to reach the gateway [2], [3], [4], [5]. Once a node is connected to a router far from the gateway, the maximum throughput it obtains is lower than the one achieved by a nearby node. Assuming there is no differentiation among nodes, there is no reason for the performance to be position dependent. This problem stems from IEEE 802.11 MAC protocol, which guarantees to each node within the same transmission range a roughly equal probability to access the medium. Nevertheless, it does not account for packets that must contend for the medium multiple times, which is the case in multihop networks.

Different works address the fairness issue in multihop wireless networks. Duffy et al. [6] analyze the performance of delay-sensitive applications. They derive an analytic model to verify the performance of multihop communications. They also propose a mechanism based on IEEE 802.11e to assign higher priority to overwhelmed routers, avoiding bottlenecks. In Raguin et al. [7], to speed up data packets transmissions, forwarding nodes merge ACK with RTS. Therefore, a forwarding node acknowledges a packet received while avoids contention for the next hop. Gambiroza et al. [2] propose IFA (Inter-transit access points Fairness Algorithm) to improve fairness. In IFA, each node computes the share of time it can transmit on each link to assure fairness. This requires information exchange about the load and link capacities. Wang and Kar [4] propose two algorithms to reduce unfairness using cross-layer design. They compute the optimal session rate at the transport layer according to link conditions. Dong et al. [5] propose MMFA (Max-Min Fair Allocation) and TBFA (Time-Based Fair Allocation) algorithms. The former divides the network throughput among transmitting nodes, whereas the latter divides the transmission time. In both cases, nodes run optimal algorithms and exchange information concerning throughput in MMFA and time in TBFA. Jun and Sichitiu [8] divide the forwarding and the originating traffic on multiple queues, and apply a scheduling algorithm to avoid starvation.

This work proposes a new MAC mechanism, called RouteLength-based Fairness (RLF), to deal with throughput unfairness caused by wireless multihop communications. Priority is assigned to packets depending on the number of hops they have to traverse to reach the gateway. Packets sent from nodes a high number of hops away from the gateway, named longlife packets, receive a higher priority in the MAC layer. The number of hops a packet traverses is known at the MAC layer considering a cross-layer design. Assigning priority improves fairness by mitigating the difference in throughput among mesh routers. Unlike previous work [6], [7], our mechanism gives priority to packets instead of to nodes. Therefore, nodes do not have to periodically compute the maximum throughput achievable, avoiding additional control frames containing information from other nodes [2], [5]. Additionally, in the proposed mechanism, packets are enqueued in different queues 
according to its route length. The idea is that packets with different route lengths are transmitted respecting the priority assigned. Simulation results show that RLF improves throughput fairness using CBR/UDP and FTP/TCP traffic compared to the conventional IEEE 802.11g. The performance of the RLF mechanism is even better when using a minimum contention window size higher than defined by IEEE $802.11 \mathrm{~g}$.

This work is organized as follows. Section II formulates the fairness problem. Section III presents our assumptions and the operation of RLF. Section IV shows our simulation results. Section $\mathrm{V}$ concludes this work and introduces next directions.

\section{FAIRNESS IN MULTiHop COMMUNiCATIONS}

In multihop networks, routers forward traffic originated by other nodes as well as their own traffic. Therefore, a node shares the bandwidth with forwarding traffic, and with other transmitting nodes within the same transmission range. Consequently, nodes which are far from the destination have increasing number of hops to traverse, implying higher contention, and reducing the throughput achievable. In mesh networks, this problem is more severe due to the stationary infrastructure and because most traffic is directed to the gateway. The throughput share is mainly unfair to nodes far from the gateway.

Another important issue is the queuing strategy applied. In standard IEEE 802.11, for instance, all packets are put into a single droptail queue. Assuming the chain topology depicted in Figure 1, if routers indefinitely increase the offered load, Router 1 starves the others [8]. Under overloaded conditions, the nearest router overflows its transmission queue much faster than it transmits or receives packets. Thus, upon receiving a packet, it discards regardless of the originating node. The network-aggregated throughput becomes then unfair because only the nearest node delivers its packets to the gateway. This work addresses this problem by privileging, at the MAC layer, packets originated at distant routers in detriment of packets originated at nearer ones.

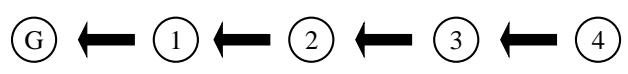

Fig. 1. Example of a 5-node chain topology.

\section{Route-Length-BASEd FAIRnESS Mechanism}

Next, we present our assumptions and the Route-Lengthbased Fairness (RLF) mechanism.

\section{A. Assumptions}

First, we consider a wireless mesh network similar to an IEEE $802.11 \mathrm{~g}$ ad hoc network. The IEEE 802.11g standard is currently the most used. Furthermore, the fairness problem in mesh networks is also seen in ad hoc networks. Both use multihop routing protocols and forwarding schemes. To aggravate the fairness problem, every router in the network originates traffic destined to the gateway. In our scenario, we consider a stationary backhaul network, where routers forward traffic from network users representing an Internet access application. In our work, we consider that every user should be provided the same service from the network and, therefore, there is no reason for one connected user to get a larger throughput.

We analyze a chain of nodes topology, such as in Figure 1. A mesh network can be approximated to a collection of chains that do not interfere [2]. This scenario is used to highlight the multihop communication effect on fairness. Also, we consider that all links in the network have the same capacity.

In the proposed mechanism, we assume that source routing is used to obtain the route length of a packet. Source routing is not needed, but it is convenient for RLF since the complete route is listed in the packet header. Also, there are different mesh routing protocols based on DSR (Dynamic Source Routing) [9]. The proposed mechanism can be easily adapted to a link-state routing protocol. In link-state protocols, each router is aware of the complete network topology. Then, finding the distance from one node to another is a matter of running Dijkstra's algorithm. In distance-vector algorithms, on the other hand, the operation of RLF becomes difficult because routers only know their neighborhood. Adapting the RLF mechanism to DV protocols needs further investigation. One possible direction is use the TTL (Time-To-Live) field to estimate the distance between two nodes.

The last assumption is the use of multiple queues, which are considered to avoid starvation. Packets are put into different queues according to their route length, to avoid contention between locally originated and forwarding packets. The multiple queues protect well-behaving nodes from starvation caused by selfish nodes. An alternative approach is to use IEEE 802.11e, which implements multiple queues and different priorities. Nevertheless, the number of queues defined in IEEE 802.11e is limited to four, which would not be suitable to our mesh topology with routers farther than four hops from the gateway. Besides, priorities are assigned at an upper layer according to application requirements.

\section{B. RLF operation}

Rather than assigning priority to nodes, the Route-Lengthbased (RLF) mechanism assigns priorities to packets according to the packet's route length. This priority is assigned by adjusting the contention window size of the packet at the MAC layer. Thus, the longer the route, the higher the priority attributed. One key characteristic of RLF is that it does not exchange control frames, avoiding overhead; and it does not compute maximum throughput, avoiding additional algorithms.

Upon receipt of a data packet, the forwarding node looks for information about the source route in the packet header. After analyzing the header, the packet is enqueued according to its route length. RLF uses one queue per route length. Hence, two nodes at the same number of hops from the gateway have their packets enqueued in the same queue. Each queue uses a FIFO/droptail algorithm, and the different queues are served in round-robin fashion.

Before forwarding a packet, the node recomputes the contention window size for this packet, according to Eq. 1. The node reduces the contention window size of the outgoing 
packet by a number given by $f(l)$. Function $f(l)$ privileges long-life packets by adjusting the contention window size according to the route length. The higher is the route length, the lower is the contention window size. In Eq. 1, $C W_{\text {new }}$ is the recomputed contention window size for the outgoing packet, $C W_{i}$ is the contention window size as defined by IEEE 802.11, and $l$ is the route length. In IEEE 802.11 MAC, $C W_{i+1}=2 \times C W_{i}+1$, where $i$ is the number of transmission attempts for a single packet, and $C W_{\min }=C W_{0}$ is the minimum contention window size. It is worth noting that the contention window size does not grow indefinitely. Instead, it remains at the maximum value until a successful transmission.

$$
C W_{n e w}=C W_{i}-f(l),
$$

where $f(l)$ is given by:

$$
f(l)=a \times\left\lfloor\frac{C W_{i}}{C W_{\min }}\right\rfloor \times l .
$$

In Eq. 2, we define $a$ is the aggressiveness index. This index is used to adjust the level of priority assigned to farther nodes. A higher aggressiveness index increases the priority. The proportion between $C W_{i}$ and $C W_{m i n}$ enlarges the $f(l)$ range, and further privileges farther nodes when packets are not successfully transmitted. Section IV demonstrates that reducing the $C W$ size of long-life packets shows a tradeoff between priority and throughput.

Supposing the 5-node chain illustrated in Figure 1, when node 4 transmits to the gateway, the source route (SR) is $4,3,2,1, G$. Upon receipt of a packet, node 2 enqueues it according to the route length. Before forwarding, node 2 computes $C W_{\text {new }}$ for the outgoing packet, which is equal to 19 assuming $a=3$, and $C W_{i}=C W_{\min }=31$.

\section{Simulation Results}

The performance of the RLF mechanism is evaluated via simulations using the network simulator 2 (ns-2) [10]. Section IV-A shows the performance of RLF using CBR/UDP traffic, and Section IV-B, using FTP/TCP traffic. We use a chain topology as depicted in Figure 1 and we vary the number of nodes in the chain. Both CBR and FTP sources send 1500byte packets. The CBR transmission rate is $3 \mathrm{Mbps}$ and the FTP sources use the TCP congestion control mechanism. In our simulations, every node produces traffic but the gateway. The gateway is the common destination of all traffic in the network, and the aggregated throughput is the sum of the throughput achieved by each node in the network.

We use the fairness index $(F)$ to evaluate RLF, where $F=\left(\sum_{i=0}^{N} T_{i}\right)^{2} /\left(N \times \sum_{i=0}^{N} T_{i}^{2}\right)$ as in [11]. In this equation, $T_{i}$ denotes the throughput of node $i$ and $N$ the number of source nodes. If $F=1$, the throughput is fairly distributed. If only one node transmits, $F=1 / N$, and the throughput is completely unfair. Additionally, we have implemented a new ns-2 module to simulate the IEEE $802.11 \mathrm{~g}$ standard. We always use RTS/CTS and the proposed aggressiveness index (a) is chosen to be 3 . In the PHY layer, the transmission rate is $54 \mathrm{Mbps}$, we use the path-loss model with $\beta=3.9$ to simulate an indoor environment [12], and we consider zero bit error rate. The reception range of all nodes is approximately $12.5 \mathrm{~m}$, which is equal to the distance between adjacent nodes in the chain, and the carrier sense range is about $75 \mathrm{~m}$. We use a confidence interval of $95 \%$.

\section{A. CBR traffic}

Figure 2(a) plots the fairness index of the chain topology with CBR/UDP sources. Note that increasing the number of nodes in the chain, RLF improves the fairness compared to conventional IEEE 802.11g. This is because packets from farther nodes receive a higher priority compared to packets originated at nearer nodes. Thus, long-life packets are transmitted faster on each hop. The use of different queues avoids starvation since they have the same probability of being served.

The decrease in fairness for longer forwarding chains occurs because the priority assigned to farther nodes by the proposed mechanism starts to produce a high collision probability. To privilege long-life packets, we reduce their contention window size. For example, if we use $C W_{\min }=31, C W_{\text {new }}$ becomes 4 assuming a 9-hop route and an aggressiveness index $(a)$ equal to 3 . Besides the collision probability, spatial reuse can further increase unfairness. In our simulated scenario, when there are more than eight nodes in the chain, the last and the first node can transmit simultaneously. Nevertheless, when the nearest node transmits, it prevents the nodes in the chain from forwarding packets. Again, the nearest node is benefited decreasing the fairness index, as seen in Figure 2(a). To cope with this problem, we have analyzed different $C W_{\min }$ values. Using higher $C W_{\min }$ sizes, the privilege of long-life packets is increased because the range of $f(l)$ is larger. Furthermore, increasing $C W_{\min }$ reduces the probability of simultaneous transmissions since the nearest node must wait longer to access the medium compared to the farthest node. This allows intermediate nodes to transmit their own packets and also forward received ones. Figure 2(a) shows that higher $C W_{\min }$ values improve fairness overcoming the collision probability and the effect of spatial reuse. The results show that compared to $802.11 \mathrm{~g}$, the fairness index increases from 0.3 to 0.95 using RLF with $C W_{\min }=255$ in a 10 -node chain. For $C W_{\text {min }}=31$, RLF reaches $F=0.45$, which is also better than $802.11 \mathrm{~g}$. Figure 2(a) also shows that depending on the number of nodes and $C W_{\min }$, the fairness index remains close to one.

The tradeoff of RLF appears on the aggregated throughput, as seen in Figure 2(b). Note that IEEE 802.11g achieves the highest aggregate throughput, however, it does not reflect a flat throughput distribution since it dedicates most network capacity to the first node after the gateway. The distribution of throughput among nodes in a 10-node chain is illustrated in Figure 2(e), where the $\mathrm{X}$ axis indicates chain position, with index 1 being the node closest to the gateway. The $\mathrm{Y}$ axis, on the other hand, shows the fraction of the aggregated throughput consumed by the node. It is seen that more distant nodes have few opportunities to send enqueued packets in $802.11 \mathrm{~g}$, which is not desirable. The RLF mechanism, in opposition, fairly distributes throughput among nodes. 


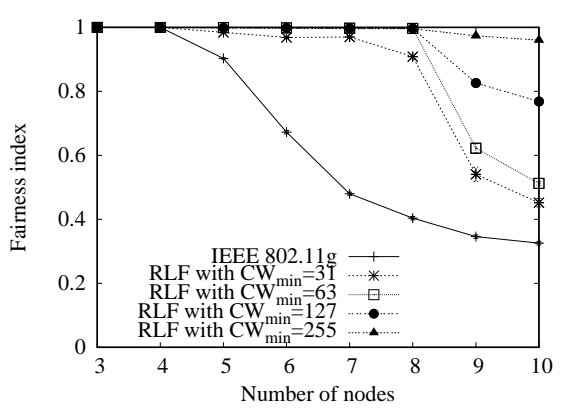

(a) Fairness index.

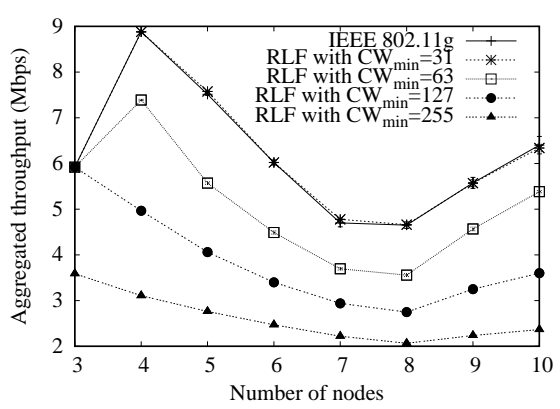

(b) Aggregated throughput.

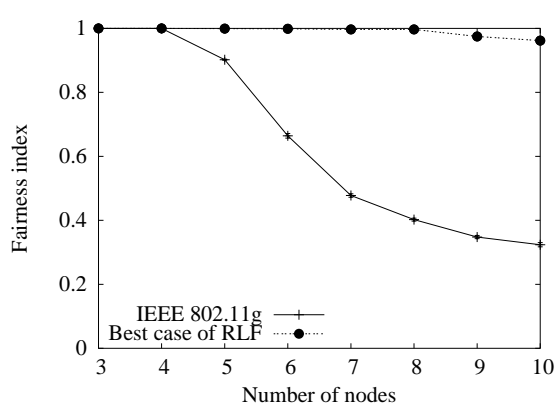

(c) Fairness index of the best-case analysis.

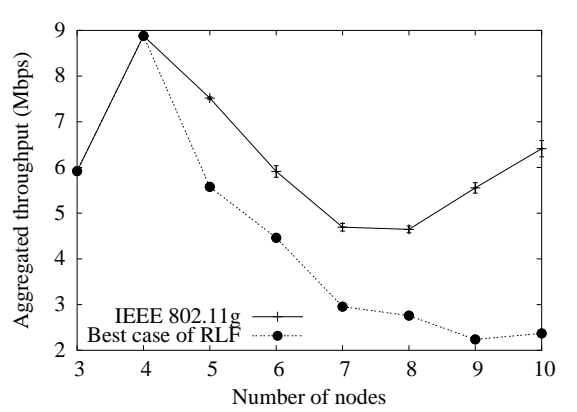

(d) Aggregated throughput of the best-case analysis.

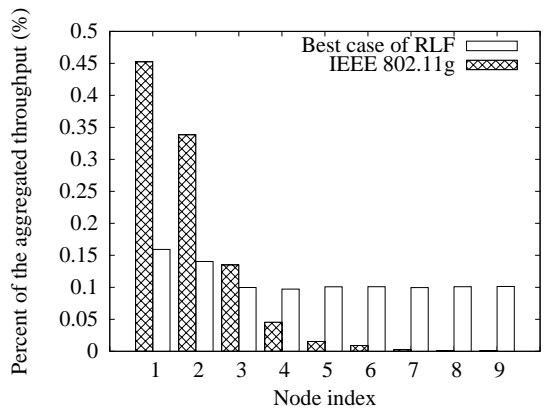

(e) Throughput distribution in a 10-node chain.

Fig. 2. CBR/UDP sources.

Figure 2(b) shows that increasing $C W_{\min }$ reduces the aggregated throughput. This happens because higher $C W_{\min }$ sizes incur in higher backoff, decreasing the maximum throughput. As with $C W_{\min }=31$ the maximum throughput was not achieved in 3-node chains, the throughput continues growing until the 4-node chain, when saturation starts. The aggregated throughput increases after 8 nodes because at this point the spatial reuse begins.

Figure 2(c) plots the fairness index of our best-case analysis. In this analysis, we use the $C W_{\min }$ that offers the best fairness index according to the number of nodes in the chain as seen in Figure 2(a). In this work, we have manually set these values because an adaptive approach needs more investigation. The values used for number of nodes, and $C W_{\min }$ are summarized in Table I. Figure 2(d) plots the aggregated throughput achieved in the best-case analysis. Observe that the fairness index keeps approximately equal to one in this case, and the aggregated throughput for few-node chains becomes as high as the throughput of IEEE $802.11 \mathrm{~g}$.

TABLE I

BEST-CASE PARAMETERS.

\begin{tabular}{|c|c|}
\hline \# nodes in the chain & $C W_{\min }$ \\
\hline \hline 3 and 4 & 31 \\
\hline 5 and 6 & 63 \\
\hline 7 and 8 & 127 \\
\hline 9 and 10 & 255 \\
\hline
\end{tabular}

\section{B. FTP traffic}

FTP sources control their transmission rates to optimize the use of the available network resources. This approach contrasts with CBR/UDP because, in the latter, sources keep transmitting at a constant bit rate, independently of the network availability.

Figure 3(a) plots the fairness index using FTP/TCP. Similarly to CBR/UDP, the fairness decreases as the number of nodes in the chain grows. Nevertheless, a fairness index close to one is already achieved with $C W_{\min }=63$. This occurs because TCP reacts to collisions by adjusting its congestion window. Therefore, by controlling the sending rate, TCP alleviates the negative effect in fairness due to collisions. Figure 3(a) shows that the fairness index is improved from 0.6 , achieved with $802.11 \mathrm{~g}$, to 1 , using RLF. Maintaining the standardized $C W_{\min }=31$, the fairness index is improved from 0.6 to 0.8 in a 10 -node chain.

Figure 3(b) plots the throughput using FTP sources. Again, the throughput of $802.11 \mathrm{~g}$ is better than the throughput of RLF, regardless the minimum contention window size. It is worth noting that, using TCP, the throughput decreases with the number of nodes. This occurs because the congestion control of TCP adjusts the transmission rate to avoid collisions, and consequently, packet losses. Especially near the gateway, where the traffic concentrates, the congestion control of TCP is more active. The mechanism adjusts the transmission rates to avoid bottlenecks, permitting that more distant nodes achieve a higher share of the available bandwidth.

Figures 3(c) and 3(d) plot the fairness index and the throughput achieved, respectively, by our best-case using the same relation between the number of nodes in the chain and $C W_{\min }$ value as shown in Table I. As with UDP, RLF can achieve a fairness index close to one, no matter the number 




(a) Fairness index.

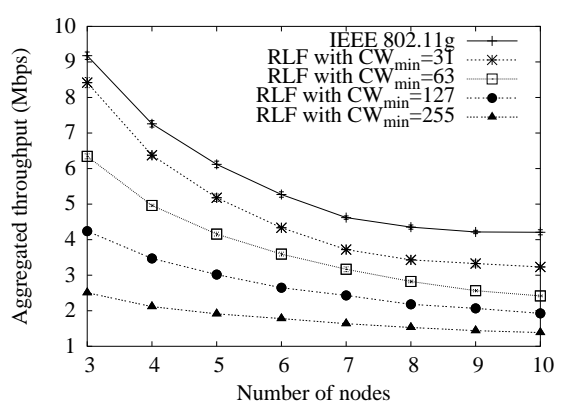

(b) Aggregated throughput.

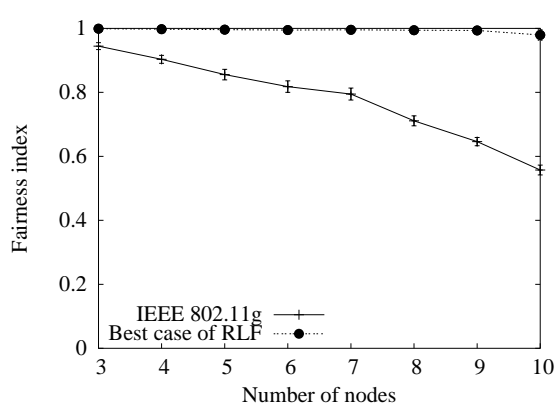

(c) Fairness index of the best-case analysis.

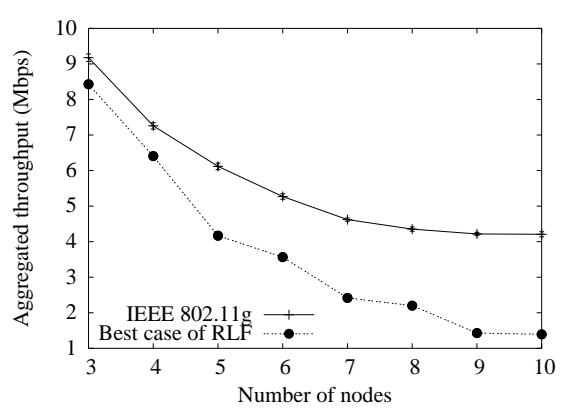

(d) Aggregated throughput of the best-case analysis.

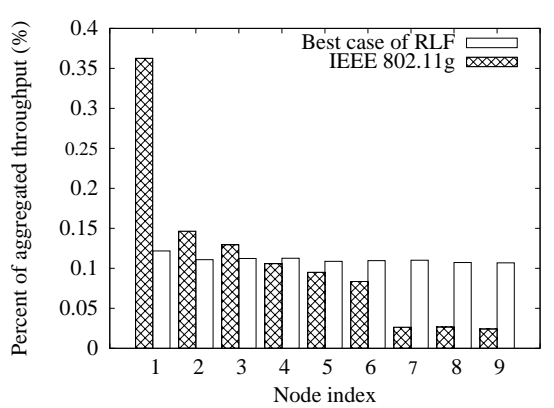

(e) Throughput distribution in a 10-node chain.

Fig. 3. FTP/TCP sources.

of nodes in the forwarding chain. Figure 3(e) shows that the throughput distribution in a 10-node chain using RLF is almost uniform also with FTP/TCP sources.

\section{CONCLUSION}

In wireless mesh networks, the stationary infrastructure and the presence of a gateway result in unfair throughput share due to multihop wireless communications. Nodes far away from the gateway tend to have a lower share of the available throughput compared to nearby ones. To improve the throughput fairness in such networks, we proposed the Route-Lengthbased Fairness (RLF) mechanism, which assigns priorities at the MAC layer according to the route length of each packet. RLF privileges long-life packets sent by farther nodes.

The simulations have shown that RLF improves fairness with the tradeoff of smaller aggregated throughput. Although the throughput of IEEE 802.11g is higher, farther nodes starve or have a lower share of the available resources. This performance is not desirable since network resources must be fairly shared among connected nodes. Our results have shown that using RLF, the fairness index reached is always higher, using CBR/UDP or FTP/TCP traffic.

As a consequence of our investigations in this work, we plan to design an adaptive mechanism to adjust the minimum contention window size according to the network conditions.

\section{ACKNOWLEDGMENTS}

This work was supported by CNPq, CAPES, COFECUB, FINEP, FUNTTEL, FAPERJ, RNP, and FUJB.

\section{REFERENCES}

[1] M. E. M. Campista, I. M. Moraes, P. M. Esposito, A. Amodei Jr., D. O. Cunha, L. H. M. K. Costa, and O. C. M. B. Duarte, "The ad hoc return channel: A low-cost solution for Brazilian interactive digital TV," IEEE Communications Magazine, vol. 45, no. 1, pp. 136-143, Jan. 2007.

[2] V. Gambiroza, B. Sadeghi, and E. W. Knightly, "End-to-end performance and fairness in multihop wireless backhaul networks," in ACM MobiCom, Sept. 2004, pp. 287-301

[3] S. M. Das, H. Pucha, and Y. C. Hu, "Symmetrical fairness in infrastructure access in multi-hop wireless networks," in IEEE ICDCS, June 2005, pp. 461-470.

[4] X. Wang and K. Kar, "Cross-layer rate optimization for proportional fairness in multihop wireless networks with random access," IEEE JSAC, vol. 24, no. 8, pp. 1548-1559, Aug. 2006.

[5] Q. Dong, S. Banerjee, and B. Liu, "Throughput optimization and fair bandwidth allocation in multi-hop wireless LANs," in IEEE INFOCOM, Apr. 2006.

[6] K. Duffy, D. Leith, T. Li, and D. Malone, "Improving fairness in multihop mesh networks using 802.11e," in International Symposium on Modeling and Optimization in Mobile, Ad Hoc and Wireless Networks, Apr. 2006, pp. 1-8.

[7] D. Raguin, M. Kubisch, H. Karl, and A. Wolisz, "Queue-driven cutthrough medium access in wireless ad hoc networks," in IEEE WCNC, Mar. 2004, pp. 1909-1914.

[8] J. Jun and M. L. Sichitiu, "Fairness and QoS in multihop wireless networks," in IEEE VTC-Fall, Oct. 2003, pp. 2936-2940.

[9] R. Draves, J. Padhye, and B. Zill, "Comparison of routing metrics for static multi-hop wireless networks," in ACM SIGCOMM, Aug. 2004, pp. 133-144.

[10] K. Fall and K. Varadhan, The ns Manual, UC Berkeley, LBL, USC/ISI, and Xerox PARC, Nov. 2006.

[11] G. Korkmaz, E. Ekici, and F. Örgüner, "A cross-layer multihop data delivery protocol with fairness guarantees for vehicular networks," IEEE/ACM Transactions on Vehicular Technology, vol. 55, no. 3, pp. 865-875, May 2006.

[12] D. Cheung and C. Prettie, "A path loss comparison between the $5 \mathrm{GHz}$ UNII band (802.11a) and the 2.4 GHz ISM band (802.11b)," Intel Labs, Tech. Rep., Jan. 2002. 